\title{
Fatigue testing and properties of hardmetals in the gigacycle range
}

Agnieszka Betzwar Kotas ${ }^{1}$, Herbert Danninger ${ }^{1}$, Brigitte Weiss ${ }^{2}$, Ken Mingard ${ }^{3}$, Jose Sanchez ${ }^{4}$, Luis Llanes ${ }^{5}$

${ }^{1}$ Technische Universität Wien, A-1060 Vienna, Austria

${ }^{2}$ Universität Wien, A-1090 Vienna, Austria

${ }^{3}$ National Physical Laboratory, Teddington, UK

${ }^{4}$ CEIT, San Sebastian, Spain

${ }^{5}$ Universidad Politecnica de Catalunya, Barcelona, Spain

Dedicated to Prof. Wolf-Dieter Schubert at the occasion of his $65^{\text {th }}$ birthday

\section{Introduction}

Hardmetals can be regarded as the most important tool materials in use today. For machining of metals, wood cutting, rock drilling etc., hardmetals, mostly WC-Co type, are indispensable. One main reason is the wide range of properties attainable by varying parameters such as binder content, carbide grain size and carbide type. Production is estimated at 75.000 tons per year worldwide.

In many applications, hard metal tools are subjected to cyclic loading. This may be interrupted cut in metal shaping, such as e.g. milling, or sawing of metals or wood, or percussion drilling of rock or concrete. The cycle numbers may vary widely but frequently exceed $10^{6}$, which means that the "very high cycle fatigue" range is entered. In addition to mechanical cycling, also thermal cycling causes damage through mechanical stresses.

The extensive studies for characterization of hardmetals included mechanical testing both in monotonic and in cyclic loading [1-9]. It is difficult to test fairly brittle materials in push-pull mode, since even a slight misalignment results in incorrect data. Therefore, bending was commonly preferred, which is easier to perform but results in a small loaded volume of the specimen. In particular cases when singular defects play a major role, testing small volumes may result in erroneous results [10].

In most cases, the tests were done up to $10^{7}$ cycles maximum. This agrees with the $\mathrm{N}$, numbers of loading cycles mostly encountered in practice. On the other hand, testing up to much higher $\mathrm{N}$, in the gigacycle range, enables elaborating the fatigue behavior and in particular the crack initiation process much more clearly. 


\section{Crack initiation in fatigue loading}

If failure of materials is described, microstructure controlled and defect controlled initiation can be distinguished. For metals this holds mostly for fatigue loading; for brittle materials such as e.g. ceramics, also monotonic loading can result in defect-controlled failure (in practice, if components and not materials are studied, of course also geometrical effects such as notches, cross holes, machining marks etc. have to be considered). "Microstructural control" is characterized by initiation at one of numerous microstructural constituents. Typical examples are cold work tool steels of 1.2379/AISI D2 type which contain a huge amount of coarse carbides. In such cases the probability that a crack initiating constituent - a large carbide or carbide cluster - is present in the loaded volume is very high [11], and therefore the scatter of the individual data is low.

Defect controlled initiation, in contrast, starts from very rare microstructural constituents. Examples are oxidic, carbidic or nitridic inclusions in high purity bearing or spring steels $[12,13]$ or slag inclusions in PM tool steels [14]. This type of fatigue failure of metals corresponds to the static failure of high performance ceramics the strength of which is typically governed by the largest defect present in the loaded volume. It can thus be derived that in case of gigacycle fatigue loading, also metallic materials show "ceramic-like" behavior.

For assessing the fatigue initiation behavior, Kitagawa-Takahashi-diagrams, also called "fatigue damage diagrams", can be used [15], as schematically depicted in Fig.1. The fatigue endurance strength (or the static strength, see [16]) is plotted against the defect size in log-log scale. Up to a certain defect size the defects are irrelevant for damage, and the strength is controlled by the microstructure. Above this critical defect size, initiation occurs at the largest defect in the loaded volume, and the fatigue (or static) strength of the material is the lower the larger the defect size is. For plotting such a diagram, the fatigue strength of the defect-free material is required as well as the „effective

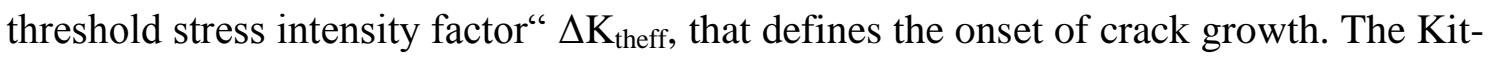
agawa-Takahashi diagram thus only depends on properties that are relevant for fatigue and does not require properties that are at least not directly correlated to fatigue, such as the hardness as e.g. in Murakami's approach [17].

However, it has to be considered that such a Kitagawa-Takahashi fatigue damage diagram holds for a given maximum loading cycle number since true fatigue limits do not exist for technical materials [18] and also since the sensitivity to singular defects increases with higher N. 


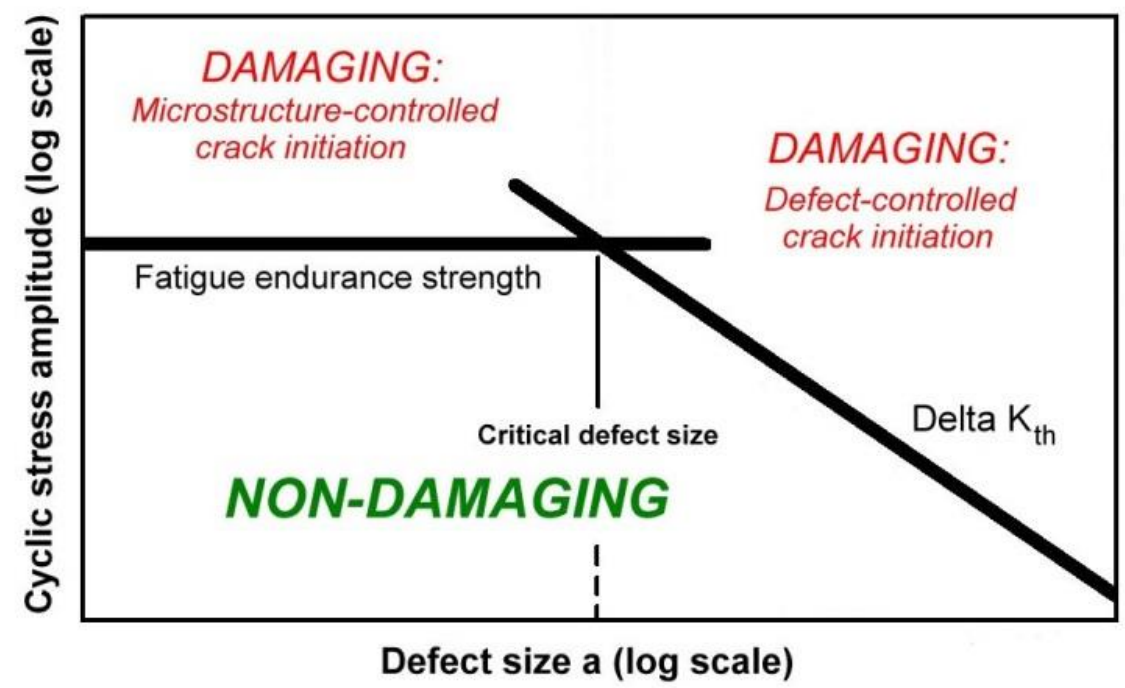

Fig.1: Kitagawa-Takahashi- fatigue damage diagram (schematic)

The present study describes the fatigue behaviour of WC-Co hardmetals in the gigacycle range, i.e. up to $\mathrm{N}>10^{9}$ cycles, ultrasonic testing being performed. Main topics were the initiation mode - microstructure- vs. defect controlled - , the existence of a true fatigue limit and the effect of parameters such as carbide grain size and binder content.

\section{Testing procedure}

The principle of the testing done here is ultrasonic resonance fatigue loading which method has been known for many decades. The development of high frequency testing methods dates back to the beginning of the $20^{\text {th }}$ century. Already in 1911 , Hopkinson [19] introduced the first electrodynamic resonance system operating up to $116 \mathrm{~Hz}$. In 1929 Jenkin and Lehman [20] used a pulsating air resonance fatigue testing system operating at frequencies up to $10 \mathrm{kHz}$. In the 1950s, Mason [21] introduced an ultrasonic fatigue testing system operating at $20 \mathrm{kHz}$. 10 years later, Neppiras [22] presented the basic principles and the mathematical equations for the design of a resonance system. Since then the ultrasonic resonance test system has been successfully applied by various groups for the determination of S-N curves, fatigue crack growth and corrosion fatigue measurements. Based on extensive studies of Stickler and Weiss [23] on the applicability of the system for fatigue testing of various materials [24-26] these authors started around 1975 to investigate the fatigue response of PM materials.

The main benefit of ultrasonic testing is the chance to get into the gigacycle range within reasonably short times. At $20 \mathrm{kHz}$, attaining $10^{10}$ cycles takes about 5 days as compared to 8 years at standard servohydraulic testers. This enables checking if a true fatigue limit exists, i.e. a horizontal branch of the S-N curve. Furthermore, the method enables finding the last remaining defects in a material, as stated above. For crack growth studies, ultrasonic testing enables very low $\mathrm{da} / \mathrm{dN}$, to $<10^{-10} \mathrm{~m} / \mathrm{cycle}$, which 
means that real threshold values are obtained. For hardmetals, ultrasonic testing should enable verifying Llanes' hypothesis that the toughness of hardmetals strongly depends on bridging effects of Co ligaments; these should be more effective in static testing that in the case of gigacycle loading [27].

In classical ultrasonic push-pull testing the specimen is fixed on one end and the other resonates freely. This is advantageous in particular for brittle materials for which clamping at both ends easily results in axiality problems. Compared to bending tests the loaded volume is much larger which is critical in case of defect-controlled initiation for which the loaded volume is a crucial parameter [10].

The testing principle is schematically shown in Fig.2 for symmetrical push-pull loading $\mathrm{R}=-1$ ); Fig.3 depicts the tester used. A transducer converts the $20 \mathrm{kHz}$ electrical signal from the generator into a mechanical oscillation which is amplified by a horn through reduction of the cross section. At the end of the ultrasonic system the specimen is fixed, usually through a thread. The entire system, including the specimen, has to be tuned such that a resonating wave is generated at approx. $20 \mathrm{kHz}$, a displacement node (i.e. a stress maximum) being generated in the gage section of the specimen. Tuning is done by adapting the length of the components according to the Young's moduli.

Compared to other fatigue testing systems, the applied stress amplitude cannot be measured directly but only through the strain amplitude, then being calculated using the dynamic Young's modulus. In practice, also the strain amplitude is measured indirectly, by a strain gage fixed at the horn which is calibrated using a calibration specimen equipped with another strain gage. Subsequently the calibration specimen is replaced by a real test specimen, and the load is applied according to the readings from the strain gage at the horn. In the studies described here, the vibration amplitude was also recorded by a Doppler vibrometer.

The indirect strain recording is required since for this variant of testing, cooling is necessary. In particular for high strength materials for which high stress amplitudes are applied, internal damping results in heat generated in the specimen, and therefore temperature control is essential. Numerous investigators prefer pulsating loads, e.g. by applying load for $100 \mathrm{~ms}$ and then cooling for $900 \mathrm{~ms}$, mostly supported by an air flow $[28,29]$. This however very much extends the testing times, and furthermore it is not clear to which degree the pulsating mode affects the fatigue behaviour. Therefore, liquid cooling was done for the present studies which proved to be highly efficient: on tool steel specimens instrumented with a thermocouple in an axial bore it could be shown that the core temperature was maximum $20 \mathrm{~K}$ above ambient. The cooling circuit is shown schematically in Fig.4a, and the cooling device in Fig.4b. 


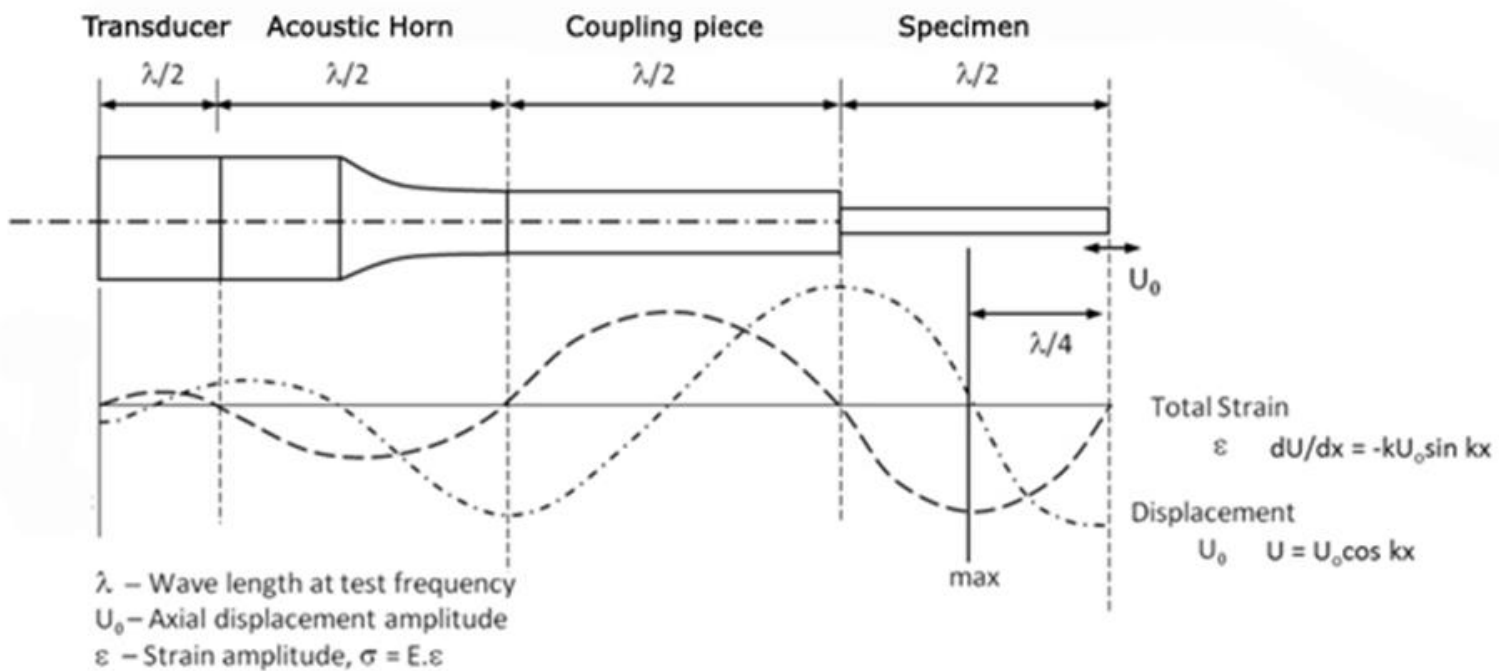

Fig.2: Ultrasonic resonance tester (schematic)

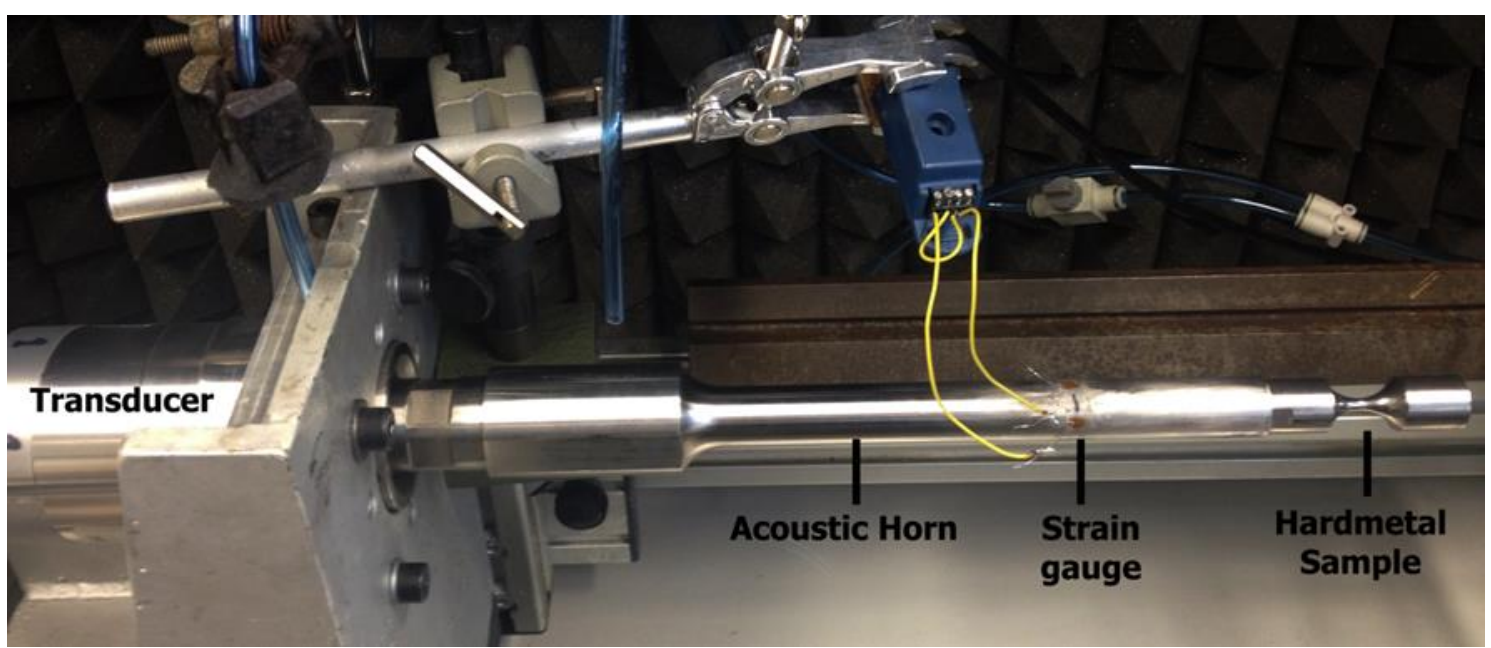

Fig.3: $20 \mathrm{kHz}$ ultrasonic resonance fatigue tester

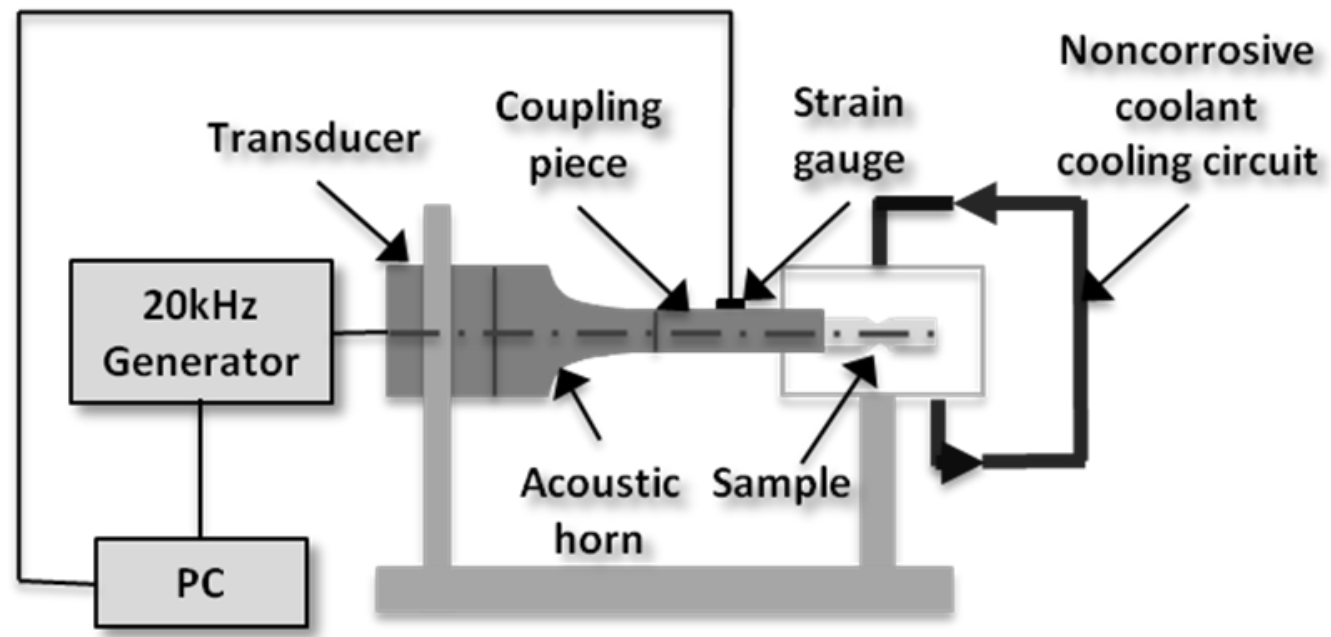


Fig.4a: Liquid cooling circuit (schematic)

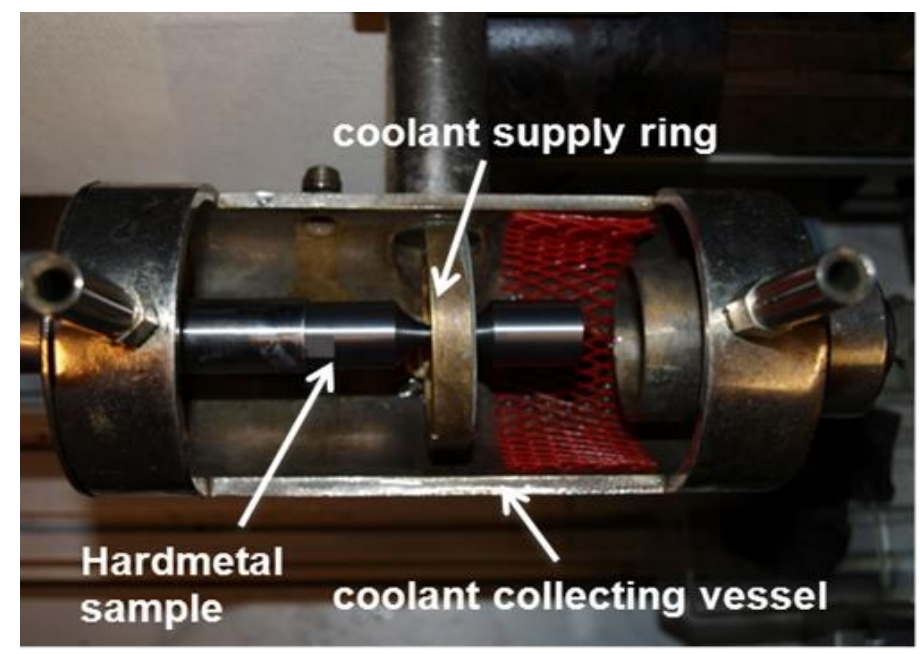

Fig.4b: Specimen cooling device

Compared to air cooling, with liquid cooling cavitation and corrosion have to be considered. Cavitation is not a problem here since the loaded surfaces in the gage area are oriented parallel to the vibration axis; only at the perpendicularly oriented end faces, traces of cavitation were observed occasionally. To prevent corrosion of the specimens during testing, the cooling liquid was water with $4 \%$ of the corrosion inhibitor Curtis Grimax CK, which is used in grinding of hardmetals.

\section{Specimen preparation}

For testing of hardmetals, an hourglass shape was selected. In order to attain the high stress amplitudes required for fracturing hardmetals with the available generator, a high amplification factor was necessary, i.e. a slim gage zone compared to bulky ends (diameters $4 \mathrm{~mm}$ vs. $18 \mathrm{~mm}$ ). The specimen geometry is shown in Fig.5a, and Fig.5b depicts the strain distribution calculated by FEM.

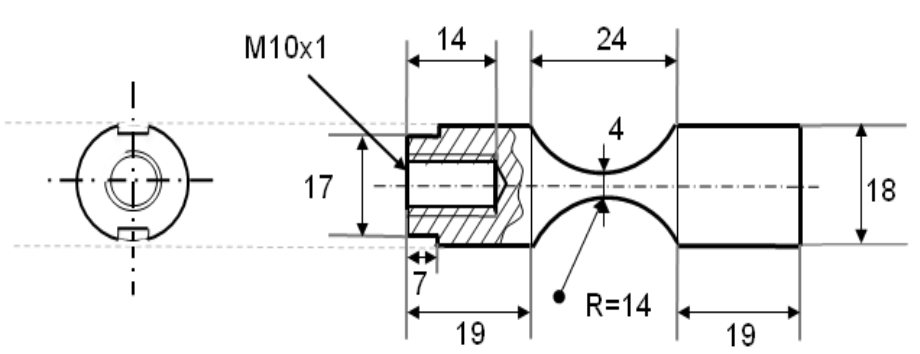

a

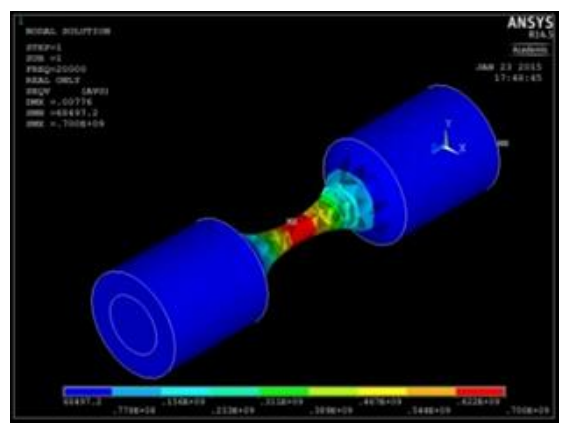

$\mathrm{b}$

Fig.5: a) Geometry of the hardmetal fatigue test specimen for $20 \mathrm{kHz}$ test;

b) FEM simulation of strain distribution in the hourglass specimen 
The specimens were manufactured by the industrial partners through the standard pressand-sinter route. Direct sintering of the hourglass specimens with the very slim gage zone was not aimed at since it was expected that porosity would remain through inhibited shrinkage. Therefore, cylindrical specimens were presintered, then an hourglass with thick gage zone was machined, and in one end a thread was cut with such oversize that sintering shrinkage was compensated for to result in an M10x1 thread after sintering. The sintered specimen was then ground to final gage section dimensions. Fig. 6 depicts a ground specimen (the thread bore is visible on the left side) as well as the surface morphology of the gage section after grinding.
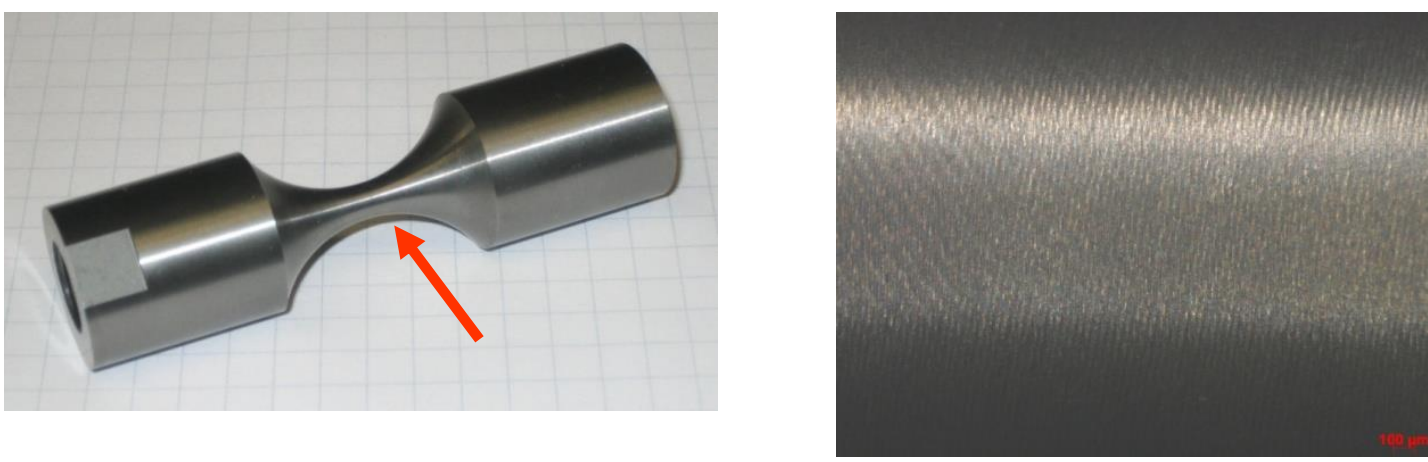

Fig.6: Test specimens ground to final dimensions and as-ground surface of the gage section

Previous studies [30] had shown that grinding and also polishing can easily result in compressive residual stresses (RS) that strongly affect the fatigue behaviour. In case of tool steels, the RS-affected surface areas can be removed by diamond polishing [30]. Therefore, the residual stress state of the hardmetal specimens was investigated by determining the RS in WC by the $\sin ^{2} \Psi$ method [31]. The results are shown in Table 1: evidently the gage section of the ground test specimen contains RS of up to -2000 MPa, markedly higher than recorded for the cylindrically ground specimens that were used for adapting the RS measuring technique. This indicates that grinding the hourglass shape is particularly stressing for the material. In contrast to the tool steels, polishing of hardmetals removed only part of the compressive RS, to about -1000 MPa. Stress relieving anneal at $800^{\circ} \mathrm{C}$ in vacuum was necessary to virtually eliminate the RS.

\begin{tabular}{|l|l|}
\hline Specimen & Residual stress (MPa) \\
\hline Cylindrical specimen $\varnothing=7 \mathrm{~mm}$, ground & $-914 \pm 25$ \\
\hline Cylindrical specimen $\emptyset=5 \mathrm{~mm}$, ground & $-710 \pm 33$ \\
\hline Fatigue test specimen, ground, gage section & $-1806 \pm 75$ \\
\hline Fatigue test specimen 1, ground and polished, gage section & $-1127 \pm 76$ \\
\hline Fatigue test specimen 2, ground and polished, gage section & $-1245 \pm 86$ \\
\hline
\end{tabular}


Fatigue test specimen, ground, polished and vacuum an$-109 \pm 23$

nealed, gage section

Table 1: Residual stresses at the surfaces of differently prepared hardmetal specimens

As stated above, during testing a resonant wave has to be generated in the system which requires adjusting of all dimensions of the system. Although the Young's moduli of the hardmetal grades used were known, in a preliminary test series, specimens with stepwise $(1 \mathrm{~mm})$ varied length were prepared from 2 hardmetal grades with different binder content. The resonance behavior was studied, and it showed that hardmetal is markedly less sensitive to the dimensions than are e.g. high alloy tool steels. All tested hardmetal specimens resonated well, and the frequency varied only slightly, between 19700 and $20500 \mathrm{~Hz}$. For the subsequent studies those dimensions were chosen that resulted in approx. $20200 \mathrm{~Hz}$.

\section{Results}

S-N curves were taken using selected hardmetal grades. As standard in fatigue testing, several runs were done for each stress level, and from the results the probability of failure was calculated. Fig.7 depicts a typical S-N (Wöhler) curve......; the low scatter of the data is clearly evident which is surprising for a low-ductility material such as hardmetal and can be taken as an indicator for microstructure-controlled initiation.

It is furthermore evident that at least up to the maximum loading cycle number of $10^{10}$ there is no fatigue limit but the graphs consistently drop with higher $\mathrm{N}$. This agrees with results obtained with other materials tested in the gigacycle range such as high alloy tool steels [11] or sintered steels [32] for which a fatigue limit was not found either, in contrast to the common notion for bcc ferrous materials but in agreement with recent studies (e.g. [18]).

Since hardmetals are known to be sensitive to corrosion,cooling was done with waterbased coolant, it was to be checked if the absence of a true fatigue limit might be caused by corrosion effects. Here however it has to be considered that the tests take fairly short time, e.g. $13 \mathrm{~h}$ for $10^{9}$ cycles. Furthermore, in some cases also internal crack initiation, "fisheye fracture", was observed for which corrosion effects should be irrelevant. For these specimens, lower stress amplitude data were recorded than in the case of initiation from the surface; if corrosion were responsible for crack initiation, the opposite should be observed, i.e. higher strength in case of internal fracture and not lower, which was however not the case. 


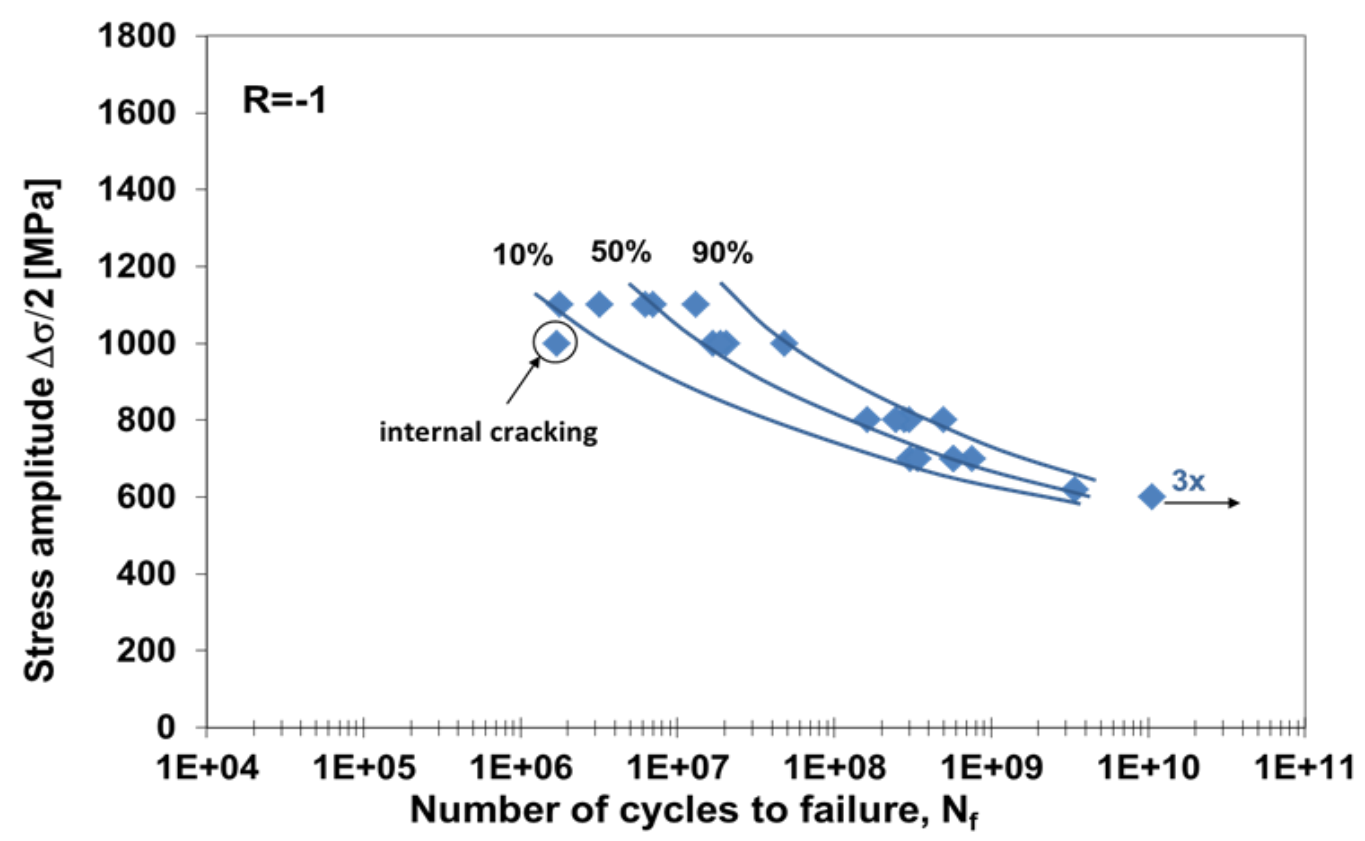

Fig.7: S-N diagram for hardmetal hourglass specimens with $6 \%$ Co and ultrafine grain size, ground, polished and annealed. push-pull, $\mathrm{R}=-1,20 \mathrm{kHz}$, water cooling with corrosion inhibitor.

For standard WC-Co hardmetals, binder content and WC grain size are the main parameters affecting the properties and thus also the application. Tough grades, such as used for milling or rock drilling, usually have binder contents $\geq 10 \%$. Fine and ultrafine grades are mostly used for metal cutting while for rock cutting and drilling rather coarse grained types are used. Knowledge of the fatigue behavior is however required for all grades.

Therefore, S-N graphs were taken for hardmetals grades with varying binder content and WC grain size, respectively. In Fig.8, graphs are depicted for grades with similar WC grain size but different binder content. Evidently the graph for the low-binder variant is shifted to higher stress level compared to the binder-rich type, which indicates that the soft binder phase adversely affects the fatigue behaviour. However, both graphs tend to converge towards higher $\mathrm{N}$

Fig.9 shows S-N graphs for medium and ultrafine grades (according to ISO) with the same binder content of $10 \%$. The shape of both graphs is virtually the same, without any fatigue limit. However, the graph for the finer grade is shifted to higher stress levels, i.e. finer WC grain size seems to be beneficial for the fatigue behavior. 


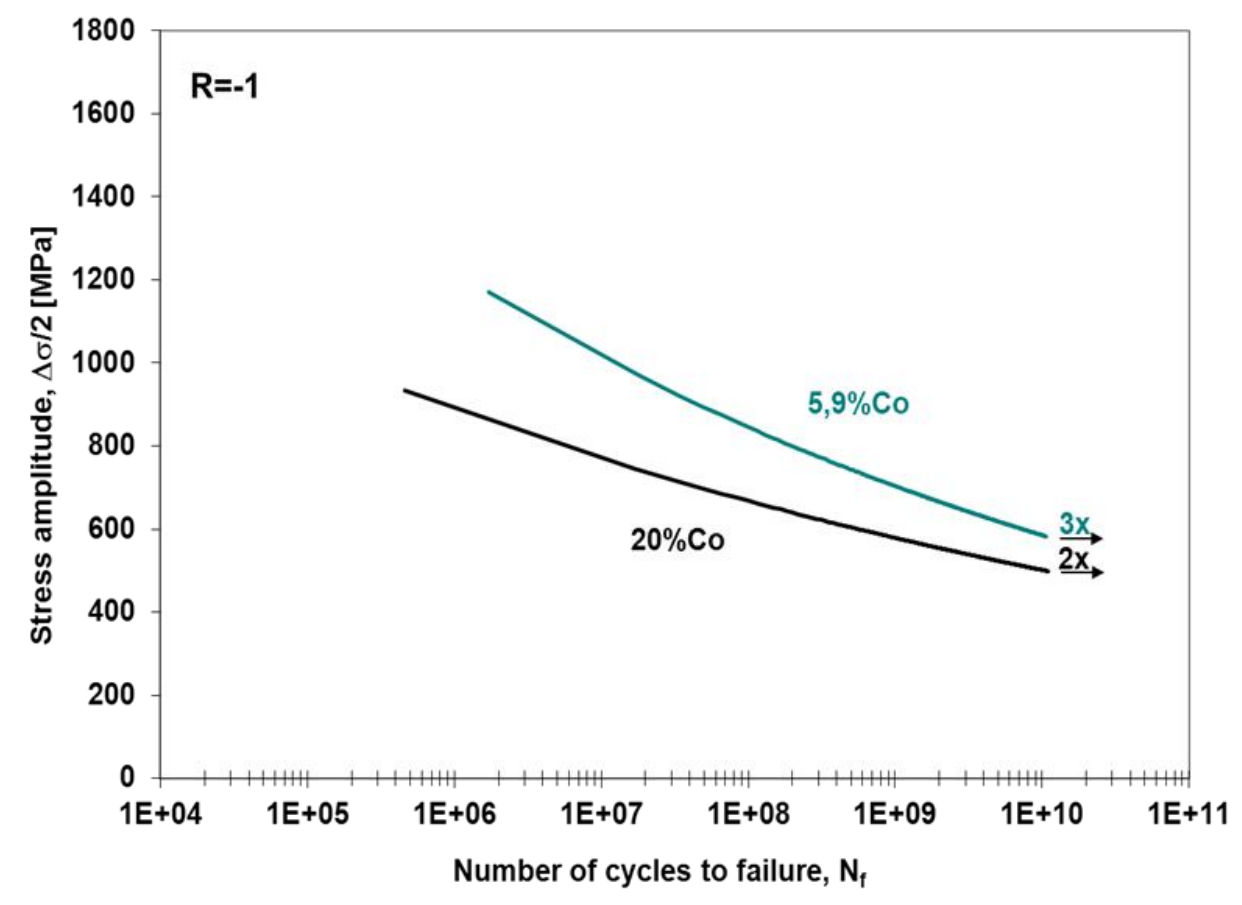

Fig.8: S-N graphs for hardmetal grades with5,9\%Co und 20\%Co, fine WC (after ISO), annealed, push-pull, $\mathrm{R}=-1,20 \mathrm{kHz}$, water cooling with corrosion inhibitor.

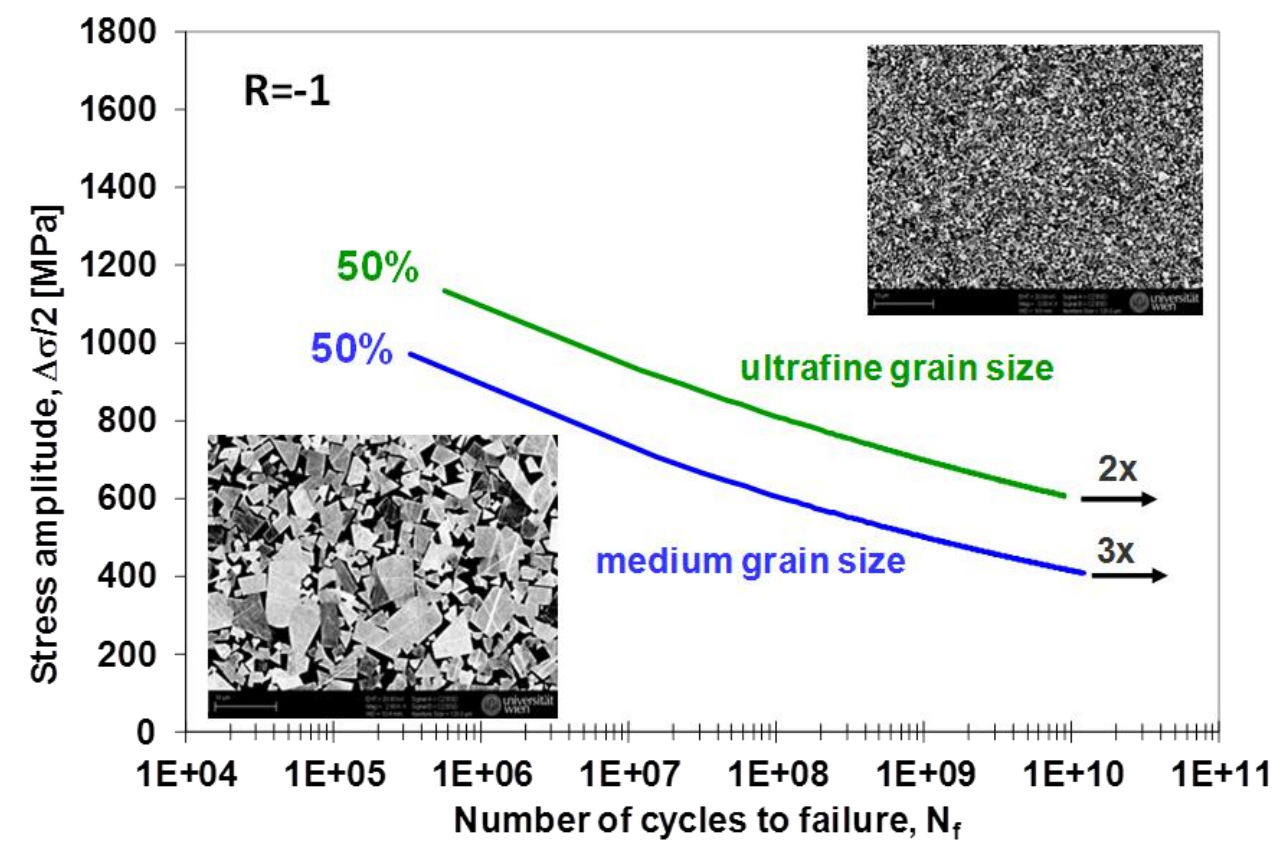

Fig.9: S-N graphs for hardmetal WC-10\%Co, different WC grain size, annealed, pushpull, $\mathrm{R}=-1,20 \mathrm{kHz}$, water cooling with corrosion inhibitor 
When comparing fatigue data obtained through ultrasonic fatigue testing with those determined through conventional techniques, there is always the question of frequency influence. In the present case this could be answered by comparison with literature data [9]. These had been measured in push-pull, $\mathrm{R}=-1$, on WC-12\%Co with $0.2-0.5 \mu \mathrm{m}$ WC grain size but at $10 \mathrm{~Hz}$. As can be seen in Fig.10, the data obtained at $10 \mathrm{~Hz}$ and 20 $\mathrm{kHz}$, respectively, can be described through a single $\mathrm{S}-\mathrm{N}$ curve, which indicates that there is almost no effect of the test frequency on the results.

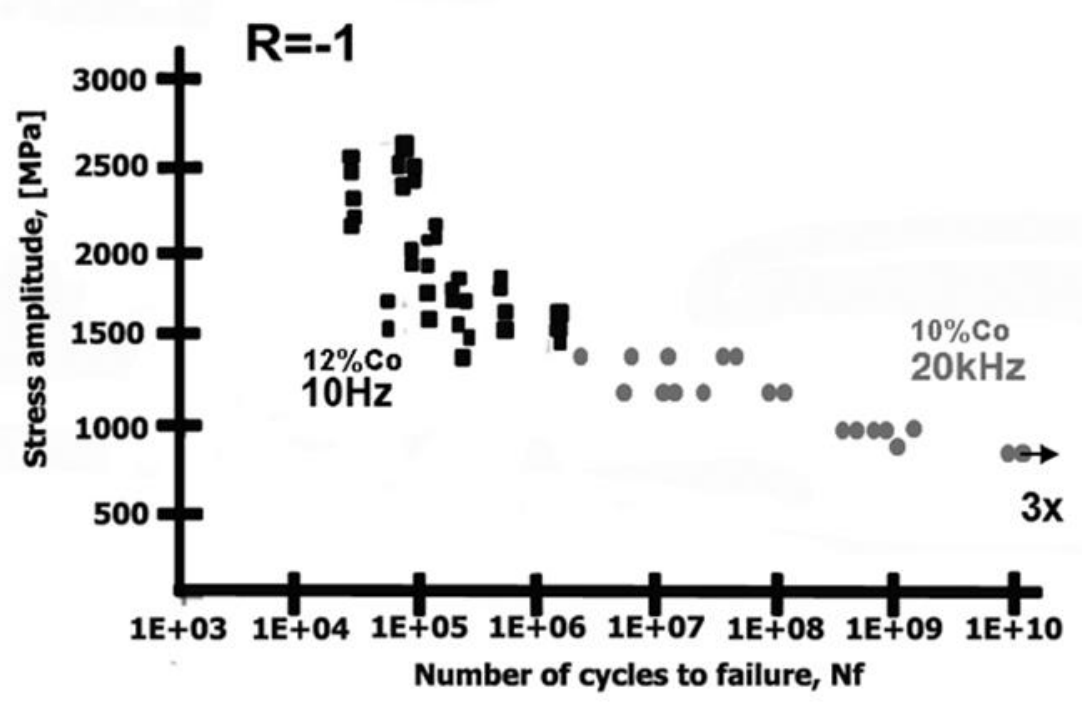

Fig.10: Comparison of S-N data obtained at different testing frequencies: $10 \mathrm{~Hz}$ (from [9]) und $20 \mathrm{kHz}$.

\section{Fractographic studies}

Investigation of the fracture surfaces, both macroscopically and by SEM, showed that crack initiation occurred almost exclusively from the surface; only in very few cases initiation from the interior, "fisheye fracture“ was observed. Examples for both cases are shown in Fig.11a, b.

For the standard case, initiation from the surface, it can be seen even with the naked eye that the area of stable crack growth, the "mirror" with smooth morphology, is only a relatively small fraction of the entire fracture surface. This is not surprising considering the fairly low fracture toughness of hardmetals. As expected, the size of the "mirror" depends on the load: the lower the stress amplitude - and the higher the resulting $\mathrm{N}$ the larger is the "mirror" (see Fig.12). 


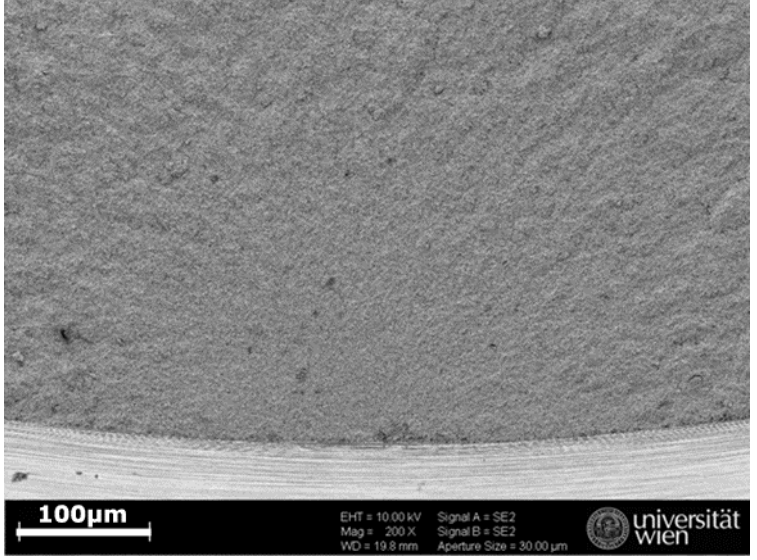

Fig.11a: Initiation from the surface

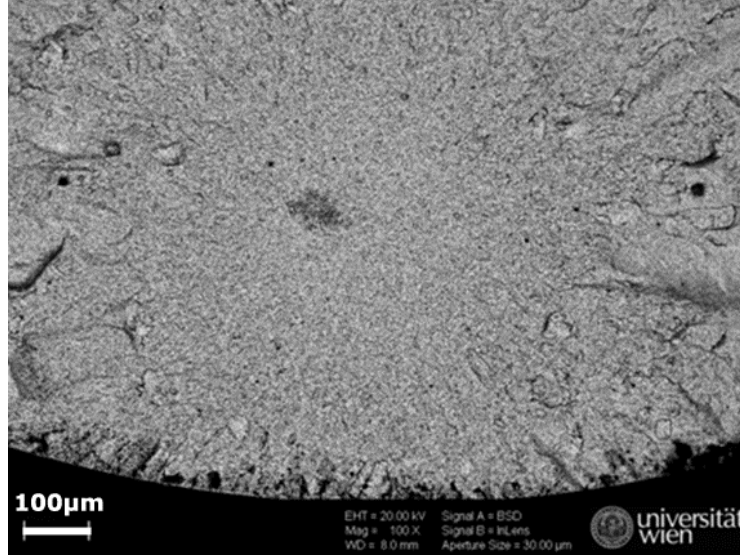

Fig.11b: Internal initiation (,fisheye“)

Fig.11: Crack initiation sites in hardmetal specimens

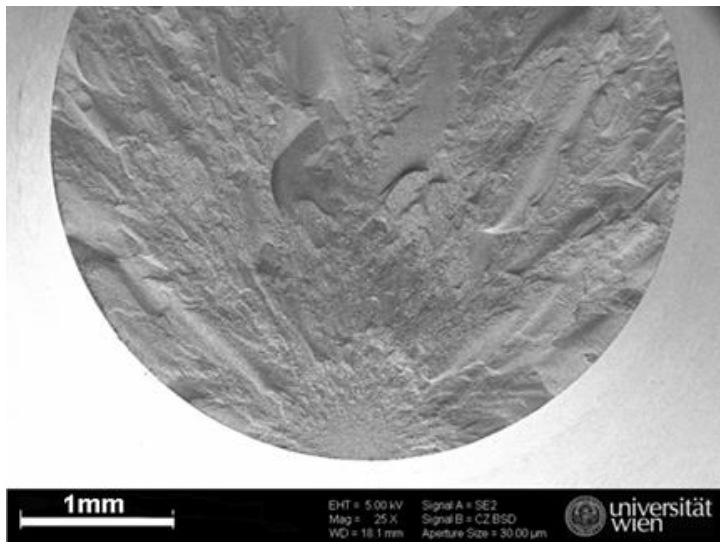

Fig12a: $\mathrm{N}_{\mathrm{f}}=6 \times 10 \mathrm{E}+05$

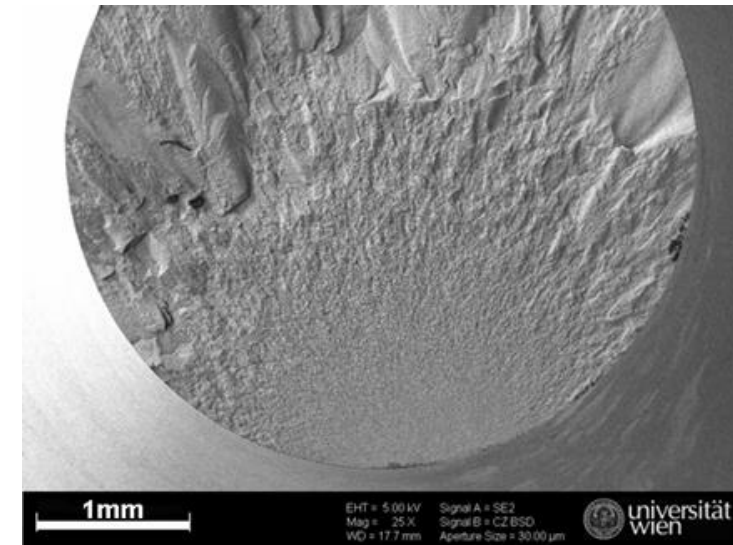

Fig.12b: $\mathrm{N}_{\mathrm{f}}=9 \times 10 \mathrm{E}+09$

Fig.12: Fatigue fracture surfaces of hardmetals tested at different stress amplitudes

When the fracture surfaces are scanned from the initiation site towards the final fracture area, significant differences are evident, in particular regarding the binder. Near the initiation site there are no signs of plastic deformation, but there is plane fracture, probably caused by failure of the interface at a large WC grain [33] (Fig.13a). At about $100 \mu \mathrm{m}$ distance from the initiation site, there is already some fatigue induced plasticity of the binder (Fig.13b), and in the final fracture area, plastic deformation of the binder is clearly evident, indicated by ridges and dimples (Fig.13c). 

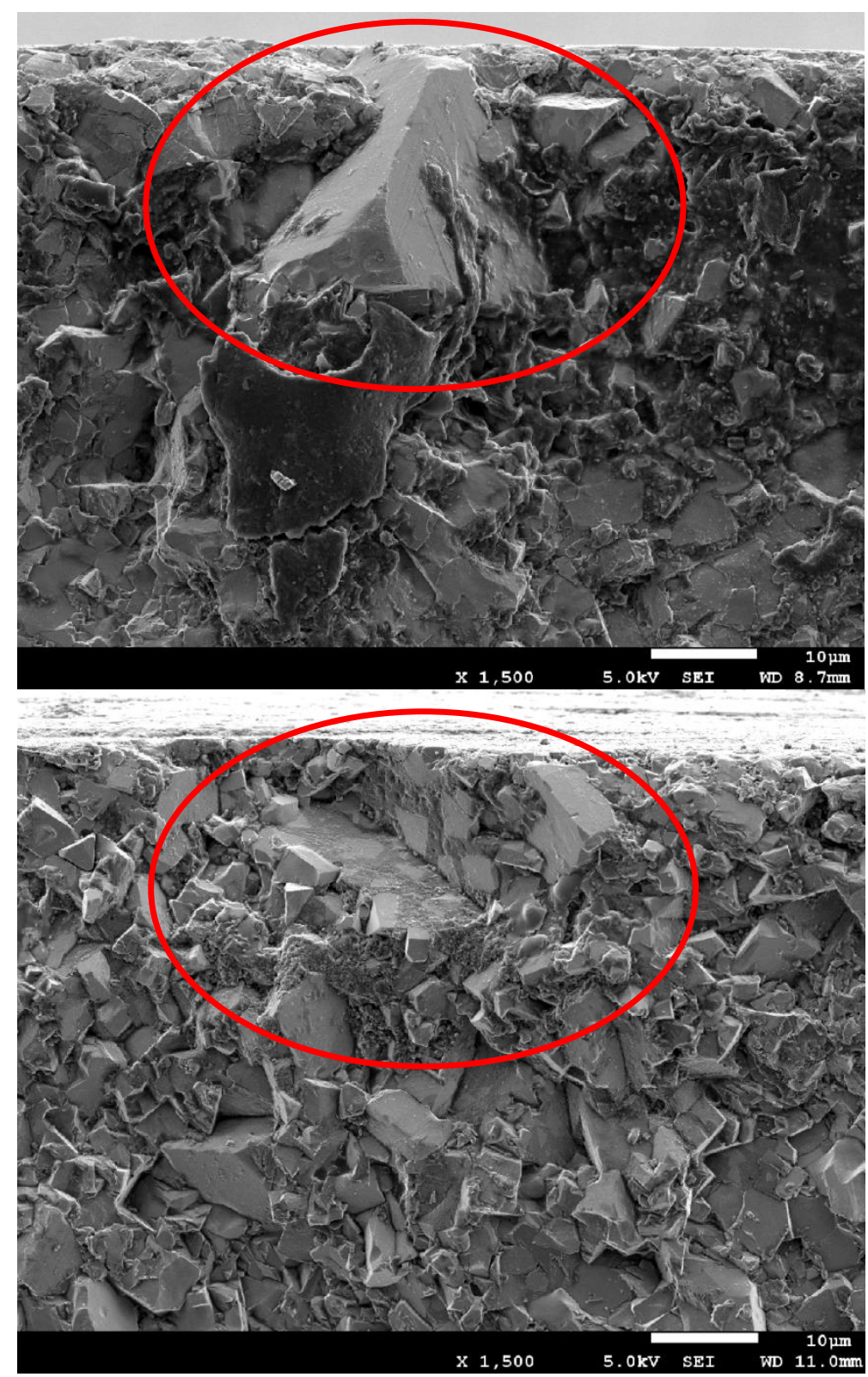

Fig.13a: Initiation site: coarse WC grain (top) and opposing fracture surface (bottom) with crystal facets 


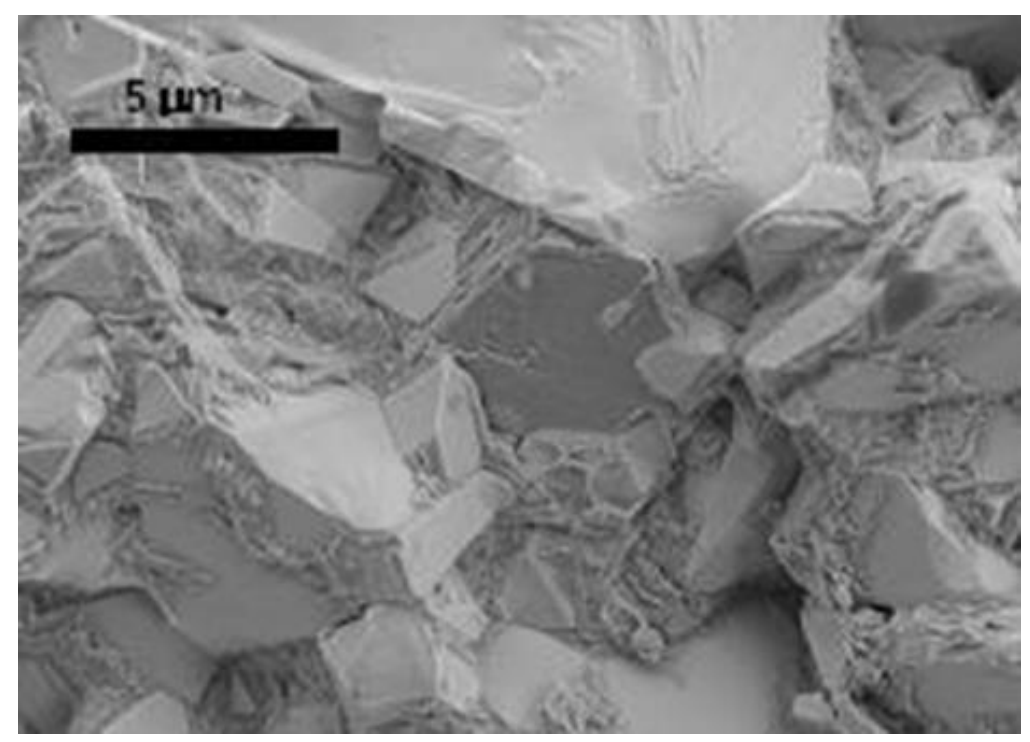

Fig.13b: „mirror“, approx. $100 \mu \mathrm{m}$ from initiation

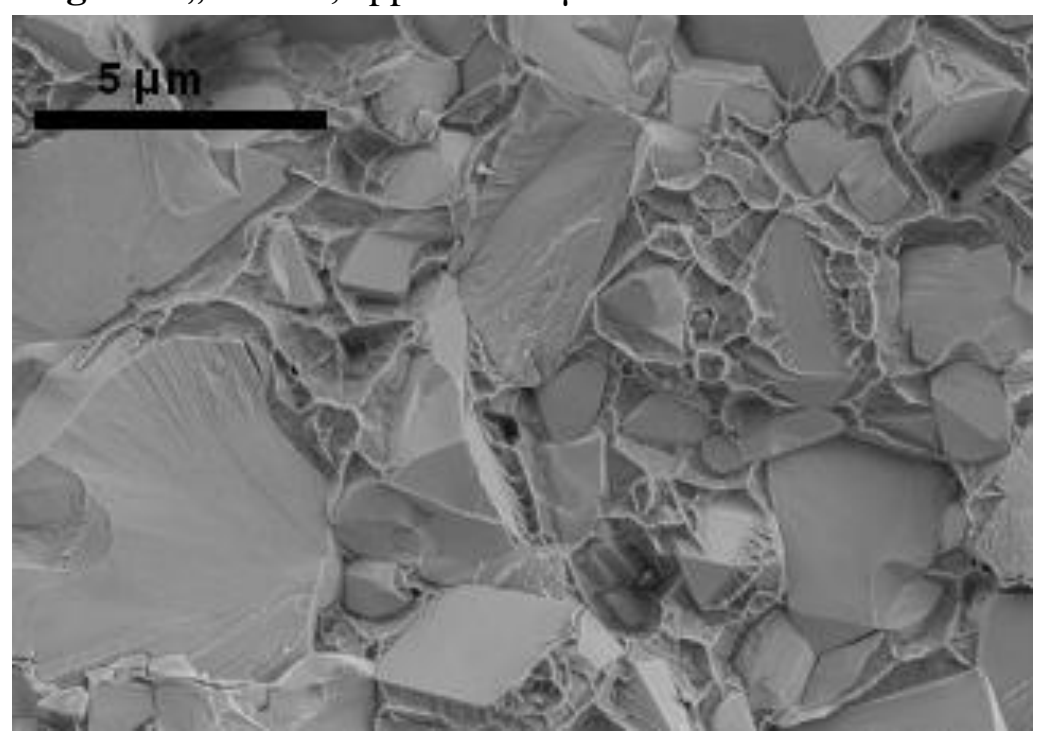

Fig.13c: final fracture

Fig.13: Fracture morphology of WC-10\%Co, medium WC grains, at different locations of the fracture surface

Generally it showed that identifying the exact crack initiation site is difficult in particular with the ultrafine grades; here it is hardly discernible from which location in the microstructure fatigue cracking has started (see Fig.14a). With coarser grades, e.g. medium and coarse variants, in contrast, it can be stated that the initiation is linked to coarse WC grains oriented such that in the fracture surface a "roof-like" structure can be seen, see Figs.13a and 14b. This indicates that cracking starts by decohesion of the binder from larger WC facets; thus the threshold is locally exceeded, and the crack propagates. It may be assumed that such decohesion effects may also occur with smaller WC grains that are oriented accordingly, but that the cracks generated are too small to propagate. 


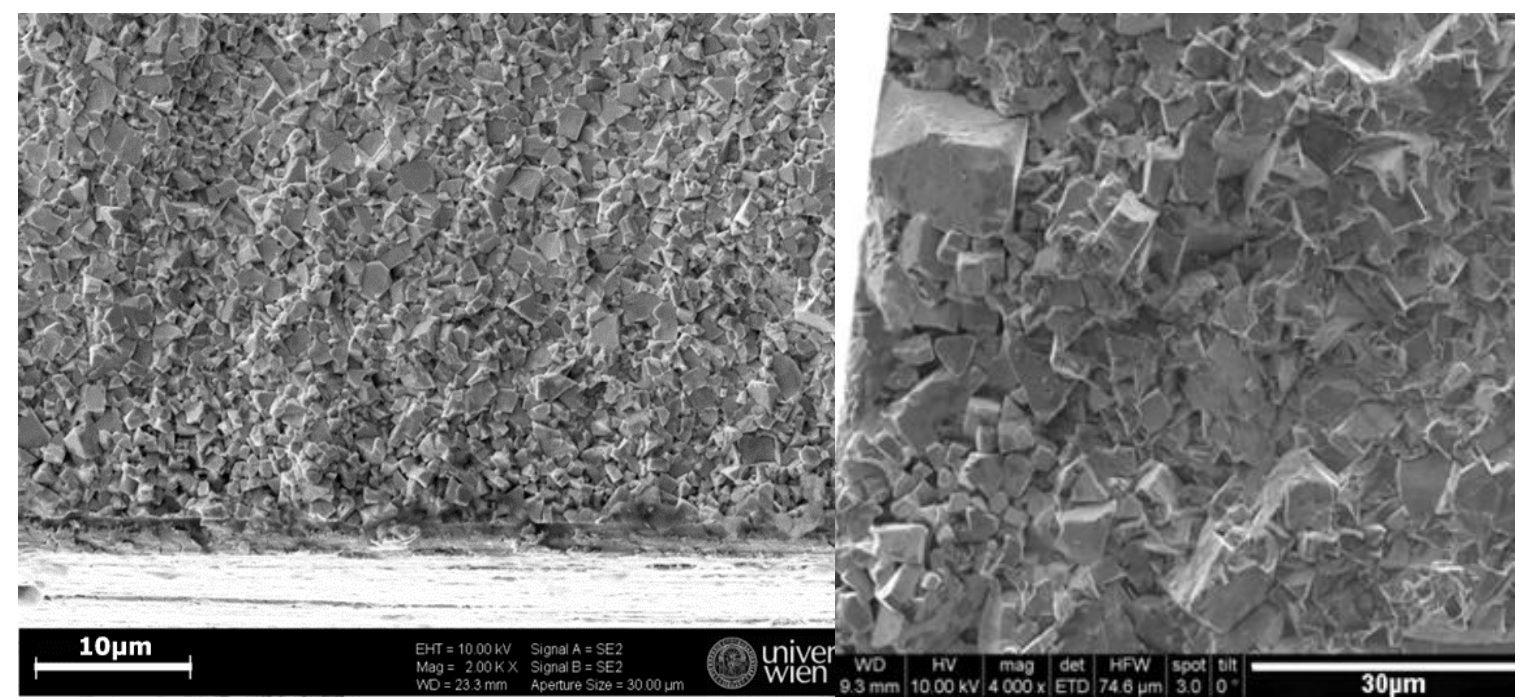

Fig.14a: WC-6\%Co- fine grade

Fig.14b:WC-10\%Co,medium grade

Fig.14: Crack initiation area in hardmetals with varying WC grain size

From these results it can be concluded that the largest WC grains or, strictly speaking, the largest plane WC crystal facets are the sites of crack initiation, in particular if for a WC larger grain two such facets meet "roof like" that are angled approx. $45^{\circ}$ to the loading axis. Since the size distribution of the WC grains in the hardmetals tested, in particular in those with mean grain size $>1 \mu \mathrm{m}$, it is highly probable that such grains are present in the microstructure. In general, those of them will initiate cracks that are close to the surface. As shown already in [34], surface or near-surface defects in a material are particularly damaging. I.e. if a large number of potentially damaging microstructural constituents are present, preferential initiation at the surface is expected, as observed here with the hardmetals.

This implies that in case of gigacycle fatigue loading hardmetals fail through microstructure-controlled initiation and not defect-controlled initiation being triggered by the numerous large WC grains. This is also the reason for the low scatter of the individual data which is typical for microstructure-controlled failure as encountered e.g. for the wrought cold work tool steel 1.2379/AISI D2 in which initiation was also caused by the largest carbides or carbide clusters. The difference is however that in the steels, crack initiation is caused by cleavage of the carbides while in the hardmetal it is interfacial failure, i.e. decohesion of the binder from large facets. It must be regarded as surprising that with low toughness materials such as hardmetals, microstructure-controlled failure occurs while with much tougher PM tool steels defect-control holds. Apparently the purity of current hardmetal grades is so high that singular defects such as pores or CaS inclusions are no more present since such defects would be revealed clearly by gigacycle fatigue loading $[12,13]$. 
For the hardmetal grade with $10 \%$ binder and medium grain size the critical defect size was estimated based on the Kitagawa diagram (Fig,1). The input data were the fatigue endurance strength at $10^{9}$ cycles as well as the effective threshold $\Delta \mathrm{K}_{\text {theff }}$. The following relationship was used:

$\Delta \mathrm{K}_{\mathrm{theff}}=\Delta \sigma \times \mathrm{F} \times(\mathrm{a})^{1 / 2}$

the geometry factor $\mathrm{F}$ being taken as 1.24 for a semicircular crack [35]. The endurance strength is $500 \mathrm{MPa}$ for this material, and $\Delta \mathrm{K}_{\text {theff }}$ was assumed as $2.5 \mathrm{MPa}^{1 / 2}[36,37]$. Calculation resulted in a critical defect size of $16 \pm 3 \mu \mathrm{m}$ is in good agreement with the experimental observations of crack initiation on similarly sized WC grains, see Figs.13a, 14b.

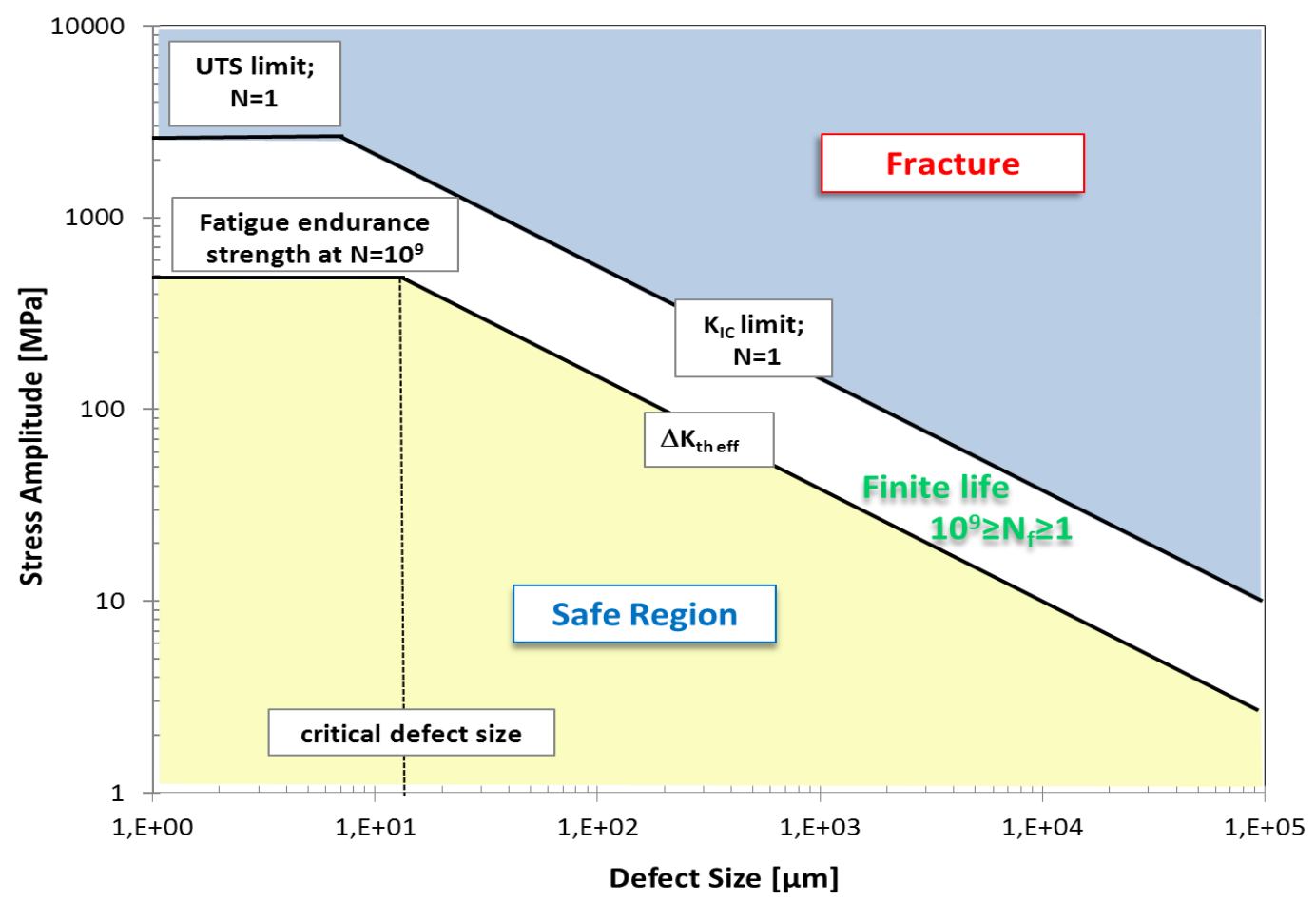

Fig.15: Fatigue life map - damage diagram- for hardmetal with 10\%Co and medium grain size

This can also be deduced from the so called "damage diagram" as shown in Fig.15 where an apparent safe region is bounded by the fatigue endurance strength at $10^{9}$ cycles and the effective threshold stress intensity factor range $\Delta K_{\text {theff. }}$ As suggested by [16] another limiting region of the diagram can be defined by conditions such as the ultimate tensile strength (or bending strength) and the fracture toughness $\mathrm{K}_{\mathrm{IC}}$, where fracture occurs in only one cycle (here the data for Fig.15 were taken from Ref [9] for a similar material). In consequence this "extended damage diagram" is demonstrated in the Fig. for a hardmetal with $10 \%$ Co binder and medium grain size, indicating the vari- 
ous fatigue life regions- safe, final life $10^{9} . \geq \mathrm{N}_{\mathrm{f}} \geq 1$ and fracture regions at higher stress levels and/or larger defect size.

\section{Conclusions}

- Gigacycle fatigue testing of hardmetals through ultrasonic resonance enables test runs up to $10^{10}$ cycles within fairly short time; thus, the existence of a true fatigue limit can be checked, and the role of singular defects can be investigated

- The testing technique is demanding, and specimen manufacturing is tricky and laborious, in particular for hardmetals

- Compressive residual stresses at the surface are easily introduced by grinding and cannot be eliminated by polishing but only by a stress relieving anneal.

- During testing, specimen cooling has to be afforded to avoid heating by damping effects. Liquid cooling using a mix of water and a corrosion inhibitor proved to be highly effective here.

- S-N graphs with surprisingly low scatter could be obtained; with finer WC grain size and lower binder content, the graphs are shifted to higher stress levels.

- Crack initiation occurs microstructure- and not defect controlled. The initiation sites are coarse WC grains generally located at or near the surface; at their larger facets, decohesion of the binder arises.

- This indicates that the hardmetal grades used are highly pure, with regular microstructure without pronounced singular defects.

- With a so-called "extended damage diagram", which we described in terms of the applied stress and flaw size, the fatigue life of hardmetals can be deduced, allowing to predict the risk of premature failure.

\section{Acknowledgement}

This work was carried out within the project „Ultrasonic Fatigue Testing of Hardmetals (UFTH)“", coordinated by the der European Powder Metallurgy Association (http://www.epma.com/projects) and financed by the following industrial partners:

CERATIZIT, Durit, Element Six, Hartmetall AG/HartmetallEstech AG, Hilti, Kennametal, Sandvik, Unimerco.

\section{References:}

[1] Exner HE., Int Met Rev; 4:149-73 (1979)

[2] Roebuck B., Int J Refract Met Hard Mater;13:265-79, (1995)

[3] Gee M., Gant A., Roebuck B., \&Mingard K. P., In V. K. Sarin, Luis Llanes\& Daniele Mari (Eds.), Comprehensive Hard Materials, Volume 1: Hard-metals. UK: Elsevier Ltd. Oxford (2014) 
[4] Roebuck B., Almond, E. A. International Materials Review, 33, 90-110 (1988)

[5] Shatov A.V., In V. K. Sarin, Luis Llanes\& Daniele Mari (Eds.), Comprehensive Hard Materials, Volume 1: Hardmetals. UK: Elsevier Ltd. Oxford (2014)

[6] Llanes L, Anglada M, Torres Y, In V. K. Sarin, Luis Llanes\& Daniele Mari (Eds.), Comprehensive Hard Materials, Volume 1: Hardmetals. UK: Elsevier Ltd. Oxford, (2014)

[7] Schleinkofer U, Sockel HG, Gorting K, Heinrich W. Mater SciEng.A;pp. 209:313 (1996)

[8] Anhai Li et al., Materials anddesign, 45, 271-278, (2013)

[9] T. Klünsner, S. Marsoner, R. Ebner, R. Pippan, J.Glatzle, A. Puschel; Procedia Eng (2010);

[10]R. Danzer: Proc. 12th Int. Plansee Seminar, Hrsg. H.Bildstein, H.M.Ortner. Reutte, Vol.2, 183 (1989)

[11] Ch. Sohar, Springer Theses, Heidelberg-Dordrecht-London-NewYork, (2011)

[12] Y. Furuya, S. Matsuoka, T. Abe; Met. Mat. Trans. A 34A, 2517 (2003)

[13] Y. Furuya, S. Matsuoka, T. AbeMet. Mat. Trans. A 35A, 3737 (2004)

[14] C.Sohar, A.Betzwar-Kotas, C.Gierl, H.Danninger, B.Weiss: Int. J. Mat. Res. 101 (2010)

[15] H. Kitagawa, S. Takahashi: Proc. 2nd Int. Conf. Mech. BehaviourofMetals, Boston MA; ASM, Metals Park OH, 627 (1976)

[16] Christodoulou L, Larsen JM: JOM 56 No.3, 15 (2003)

[17] Y. Murakami, N.N. Yokoyama, J. Nagata: Fatigue Fract. Eng. Mater.Struct., 25, $735(2002)$.

[18] C.M.Sonsino: Konstruktion No.4, 87 (2005)

[19] B.Hopkinson: Proc. R. Soc., A $86,101,(1911)$

[20]C.Jenkin and G. Lehman, Proc. R. Soc., 83, (1929)

[21] W.P.Mason, Ultrasonics, van Nostrand, N.Y. (1950)

[22] E. A. Neppiras, P Proc. ASTM, 691, (1959)

[23] R. Stickler et al., Ultrasonic Fatigue, AIME, Seven Springs USA, (1981)

[24] B. Weiss et al.: Metall 34, 636, (1980)

[25] H. Danninger et al., J. Mat.ProcessTechn., 179-184, (2003)

[26] A. Hadrboletz et al., Int. Mat. Rev., 42, 43-53, (1997)

[27] L.Llanes: EPMA, Winterev workshop,(2009),

[28] H.Mayer: Int. J. Fatigue;28(11):1446-55, (2006)

[29] C. Bathias, P. Paris, Gigacycle fatigue in mechanical practice M. Dekker, (2005)

[30] Ch. Sohar, A. Betzwar-Kotas, C. Gierl, B. Weiss, H. Danninger: Materialwissenschaft und Werkstofftechnik, 39 No.3, 248 (2008).

[31] A.J. Perry, et al. Surf.Coat. Technol., 81, 17-28 (1996)

[32] H. Danninger, C. Xu, G. Khatibi, B. Weiss, B. Lindqvist: Powder Metallurgy, 55 No.5, 378 (2012)

[33] K.P. Mingard, B. Roebuck, UFTH Project, Final Report (2013)

[34] W. Betz,W. Track,109th AIMEMeeting Las Vegas (1980)

[35] D. Spoljaric, B.Weiss, H. Danninger, PM 94, Volume 2, Paris (1994)

[36] L. Llanes, Y Torres, M Anglada; ActaMaterialia, Volume 50, Issue9, 2381 (2002)

[37] B.Weiss, R.Stickler; Proc. $11^{\text {th }}$ Int. Plansee Seminar (1985) 